\title{
Core-in-cup/liquisol dual tackling effect on azelnidipine buccoadhesive tablet micromeritics, in vitro release, and mucoadhesive strength
}

\author{
AMIRA A. RASHAD ${ }^{1}$ \\ SARA NAGEEB EL-HELALY ${ }^{2 *}$ \\ RANDA T. ABD EL REHIM ${ }^{2}$ \\ OMAIMA N. EL-GAZAYERLY2 \\ ${ }^{1}$ Mepaco-Medifood Pharmaceutical \\ Company, El Sharkia, Egypt \\ ${ }^{2}$ Department of Pharmaceutics \\ and Industrial Pharmacy, Faculty \\ of Pharmacy, Cairo University \\ Cairo, Egypt
}

Accepted November 30, 2018 Published online December 14, 2018

\begin{abstract}
Reduced bioavailability of azelnidipine is related to its poor aqueous solubility and extensive first-pass metabolism, which hinder its efficacy. These problems were addressed by implementing (1) a liquisol technique for promoting the dissolution rate in a controlled-release manner and (2) a core-in-cup buccoadhesive drug delivery system as an alternative to the oral route. A $3^{3}$ factorial design was used to study the effects of polymer type (sodium carboxymethyl cellulose (CMC Na), chitosan, or Carbomer P940) concentration (5, 10 or $15 \%$ ) and preparation technique (simple mix, liquisol or wet granulation) on the dissolution and mucoadhesion of core-in-cup azelnidipine buccoadhesive tablets. Tablet micromeritics, swelling index, mucoadhesive strength and in vitro release were characterized. Statistical analyses of these factors showed significant effects on the studied responses, where $\mathrm{F} \# 16$ prepared by the liquisol technique and containing $15 \% \mathrm{CMC}$ Na was chosen with an overall desirability of 0.953 .
\end{abstract}

Keywords: azelnidipine, liquisol, core-in-cup, buccoadhesive tablets, tablet micromeritics, in vitro release

Azelnidipine (AZL), a dihydropyridine calcium channel blocker, stimulates peripheral vasodilation by blocking calcium channels in vascular smooth muscle cells without increasing the heart rate. Poor bioavailability of AZL, which has the biopharmaceutics classification system (BCS) rating of class II and the molecular weight 582.65, is related to its poor aqueous solubility (less than $1 \mathrm{mg} \mathrm{mL}^{-1}$ ) and high lipophilicity partition coefficient ( $\log P=7$, octanol/Sorensen's buffer pH 7.4) (1, 2). Moreover, AZL undergoes extensive first-pass metabolism by cytochrome P450 (1), which hinders its efficacy in pharmaceutics. Conventional drug delivery has some limitations, viz., increased chances of missing the drug dose when frequent administration is necessary and an atypical plasma concentration time profile making it difficult to attain a steady state.

\footnotetext{
*Correspondence, e-mail: sara.elhelaly@pharma.cu.edu.eg, http://orcid.org/0000-0001-6382-0995
} 
Accordingly, these problems have been addressed using the liquisol technique, primarily to enhance the solubility and promote the dissolution rate of AZL. First, the drug is solubilized in suitable solvents before being converted into free-flowing compressible powdered mixtures by blending with the selected carrier and coating materials (3). Second, core-in-cup (CIC) buccoadhesive drug delivery is used as an alternative to the oral route to bypass AZL first-pass metabolism. CIC offers a safe and easy method for drug utilization, since the drug action can be promptly terminated by removing the CIC buccoadhesive tablet from the buccal cavity. This technique aims to control AZL release in a zero-order manner, ensuring unidirectional release and maintaining plasma concentrations within the therapeutic window (4) to maintain normal blood pressure levels. These two methodologies were chosen for their ease in scaling up and hence their suitability for commercial production.

Thus, this research studied the factors affecting both tablet mucoadhesion (to ensure full-time contact with the buccal mucosa) and in vitro drug release. A buccoadhesive drug delivery system using liquisol was formulated to enhance AZL dissolution and avoid firstpass metabolism.

\section{EXPERIMENTAL}

\section{Materials}

AZL was purchased from (Zhejiang Gaobang Pharmaceutical Co. Ltd., China), carboxymethylcellulose sodium 50-200 mPa s was purchased from (CP Kelco, USA). High-MW chitosan (310000-375000 Da) was purchased from (Sinochem Jiangsu, China). Carbomer P940 was purchased from (Aqualon, USA); Poloxamer 188 was purchased from (BASF, Germany); and Labrasol ${ }^{\circledR}$ and Transcutol ${ }^{\circledR} \mathrm{HP}$ were kind gifts from (Gattefosse, Saint-Priest, France). Cremophor ${ }^{\circledR}$ RH40 was kindly provided by (BASF SE, Carl-Bosch, Germany). Polyethylene glycol 6000 was procured from (El-Nasr Pharmaceutical Chemicals Co., Egypt). Mannitol was purchased from (Shandong Bangye Co. Ltd., China), ethyl cellulose was purchased from (FMC Biopolymer, Belgium), and magnesium stearate was purchased from (Aceto-Corp, USA). Sorenson phosphate buffer components (sodium phosphate dibasic anhydrous and sodium dihydrogen orthophosphate dihydrate) were purchased from (Loba Chemie, India). Aeropril 300 was obtained from (Degussa Ltd., Germany), high-performance liquid chromatography (HPLC)-grade acetonitrile was purchased from (Merck, Germany), and HPLC-grade methanol was purchased from (Fisher Scientific, UK). All other reagents were of analytical grade.

\section{High-performance liquid chromatography (HPLC) analyses}

AZL was quantified via a validated liquid chromatographic method using a Schimadzu instrument (UFLC Prominence 20 XR) with a UV detector for linearity, accuracy, selectivity and precision (5). A Thermo C18 column $(5 \mu \mathrm{m} ; 4.6 \times 150 \mathrm{~mm})$ was used, and the mobile phase was a mixture of (acetonitrile/methanol/water, 2:2:1) $(V / V)$, which was filtered, degassed and eluted at a flow rate of $2 \mathrm{~mL} \mathrm{~min}^{-1}$. Elution was detected at $260 \mathrm{~nm}$, and the injection volume was $20 \mu \mathrm{L}$. 


\section{Preformulation studies}

Differential scanning calorimetry (DSC)

DSC was performed on the AZL, excipients and their 1:1 $(\mathrm{m} / \mathrm{m})$ physical mixtures (PMs). The excipients tested were carboxymethylcellulose sodium (CMC Na), chitosan, Carbomer P940, mannitol, Aeropril 300, and ethyl cellulose. DSC analysis was performed using a differential scanning calorimeter (DSC-60, Shimadzu, Japan). Samples (3-4 mg) were placed in aluminium pans and heated to $10-400{ }^{\circ} \mathrm{C}$ at a rate of $10^{\circ} \mathrm{C} \mathrm{min}^{-1}$, with indium in the reference pan under a nitrogenous atmosphere. DSC was used to determine any possible physicochemical interactions between the drug entity and the excipient used in its formulation.

\section{Fourier transform infrared spectroscopy (FTIR)}

FTIR spectra of $450-4000 \mathrm{~cm}^{-1}$ of the drug, excipients and their 1:1 $(\mathrm{m} / \mathrm{m})$ PMs were determined using an infrared spectrophotometer (Fourier Transform, FTIR-84005, Shimadzu) and the potassium bromide disk technique (6).

\section{Screening the effects of different solubilizers on AZL solubility}

Equilibrium solubility of AZL was studied by preparing the PMs of AZL with five solubilizers (Poloxamer 188, Cremophore RH40, Transcutol HP, Labrasol, and PEG 6000) at three ratios (1:1, 1:3 and 1:5, $\mathrm{m} / \mathrm{m}$ ). The PMs were prepared by mixing $25 \mathrm{mg}$ of the drug with each solubilizer for $2 \mathrm{~min}$ to obtain homogeneous mixtures. To determine the solubility of AZL in these mixtures, each PM was shaken with $25 \mathrm{~mL}$ distilled water in an amber-colored glass vial, and the blank was $25 \mathrm{mg}$ of the drug with $25 \mathrm{~mL}$ water only. The PMs were then left in a shaker at room temperature $\left(25^{\circ} \mathrm{C}\right)$ for $24 \mathrm{~h}$. The samples were withdrawn, filtered and analyzed using the previously described HPLC method.

\section{Applying a $3^{3}$ full factorial design for buccoadhesive tablets}

Three factors were studied (the mucoadhesive polymer type, the polymer concentration and the preparation technique) to evaluate their influence on drug dissolution and mucoadhesion. A $3^{3}$ full factorial design was conducted yielding twenty-seven randomly performed AZL formulations. The factors studied with their respective levels and constraints are shown in Table I. The choice of factors and their respective levels was based on a literature review, field experience and preliminary studies conducted before implementing the experiment. Other variables, such as drug amount, solubilizer type (Poloxamer 188), carrier type (mannitol) and coating material (Aeropril 300) were kept constant. The responses measured were in vitro drug release after $2 \mathrm{~h}(\mathrm{Q} 2), 4 \mathrm{~h}(\mathrm{Q} 4)$, and $8 \mathrm{~h}(\mathrm{Q} 8)$ and mucoadhesive strength.

Design-Expert software (V. 7.0.0, Stat-Ease Inc., USA) was used to apply and evaluate the factorial design. Means were compared by a factorial analysis of variance (ANOVA). The significance level was set at $\alpha=0.05$. Numerical optimization was performed using a statistical program per the constraints listed in Table I. The simultaneous optimization technique, based on the use of desirability functions, was chosen to optimize the responses. All experiments and characterizations were performed at least three times. All data are presented as the mean \pm standard deviation. 
A. A. Rashad et al.: Core-in-cup/liquisol dual tackling effect on azelnidipine buccoadhesive tablet micromeritics, in vitro release, and mucoadhesive strength, Acta Pharm. 69 (2019) 381-398.

Table I. $3^{3}$ full factorial design: factors and responses

\begin{tabular}{|c|c|c|c|}
\hline \multirow{2}{*}{$\begin{array}{l}\text { Factors (independent variables) } \\
\text { A: Type of mucoadhesive polymer }\end{array}$} & \multicolumn{3}{|c|}{ Levels } \\
\hline & CMC Na & Chitosan & Carbomer P940 \\
\hline B: Concentration of mucoadhesive polymer & $5 \%$ & $10 \%$ & $15 \%$ \\
\hline C: Technique of core preparation & Simple mix & Liquisol & Wet granulation \\
\hline Responses (Dependent variables) & \multicolumn{3}{|c|}{ Constraints $^{\mathrm{a}}$} \\
\hline R1: \% drug released from the tablet after $2 \mathrm{~h}(\mathrm{Q} 2 \%)$ & \multicolumn{3}{|c|}{$30 \% \geq R_{1} \geq 20 \%$} \\
\hline $\mathrm{R} 2: \%$ drug released from the tablet after $4 \mathrm{~h}(\mathrm{Q} 4 \%)$ & \multicolumn{3}{|c|}{$60 \% \geq R_{2} \geq 40 \%$} \\
\hline R3: \% drug released from the tablet after $8 \mathrm{~h}(\mathrm{Q} 8 \%)$ & \multicolumn{3}{|c|}{$\mathrm{R}_{3} \geq 80 \%$} \\
\hline R4: Mucoadhesive strength (g) & \multicolumn{3}{|c|}{ Maximize } \\
\hline
\end{tabular}

${ }^{\text {a }}$ Constraints were set according to the international guidelines (9).

\section{Preparation of core-in-cup (CIC) buccoadhesive tablets}

\section{Core preparation}

Detailed composition of all formulations is shown in Table II. All formulations contained $8 \mathrm{mg}$ AZL. Three mucoadhesive polymers (CMC Na, chitosan and Carbomer P940) were studied at three ratios $(5,10$ and $15 \%)$. Three techniques were used to prepare the core powder mixture.

Simple mix technique (SM). - Calculated amounts of the drug, mucoadhesive polymer, Poloxamer 188, Aeropril 300, and mannitol were separately sieved using a sieve with 710$\mu \mathrm{m}$ pores. The powders were mixed using a mortar and pestle to form a dry powder mixture.

Liquisol technique (LS). - Poloxamer $188(24 \mathrm{mg})$ was melted at $55^{\circ} \mathrm{C}$, then $8 \mathrm{mg}$ AZL was added (AZL/Poloxamer 188, 1:3, $\mathrm{m} / \mathrm{m}$ ) and stirred until the AZL completely dissolved in Poloxamer 188. Mannitol was added geometrically to improve the effective surface area for dissolution and to assist the compression. Next, the coating material (Aeropril 300) was added to improve the flowability, absorb excess liquid and give a dry appearance by covering wet carrier particles. Predetermined mucoadhesive polymer concentrations were then added to the mixture. Excipients were then geometrically mixed in a mortar for $20 \mathrm{~min}$, and the final mixture was sieved using a sieve with $710-\mu \mathrm{m}$ pores.

Wet granulation technique (WG). - AZL, the polymer, Aeropril 300, and mannitol were mixed using a mortar and pestle for $20 \mathrm{~min}$ to form a powder mixture. The granulating solution was prepared by dissolving Poloxamer 188 in $95 \%$ ethanol at twice its weight. The powder mixture was granulated with the previously prepared solution and then sieved through a sieve with $850-\mu \mathrm{m}$ pores. The granules were dried in an oven at $50{ }^{\circ} \mathrm{C}$ until the moisture content of bulk granules reached 2-3\% and then sieved using a sieve with 710$\mu \mathrm{m}$ pores to form a dry powder mixture. Moisture content was measured using a moisture analyzer (Schimadzu MOC-120HEXP). 
A. A. Rashad et al.: Core-in-cup/liquisol dual tackling effect on azelnidipine buccoadhesive tablet micromeritics, in vitro release, and mucoadhesive strength, Acta Pharm. 69 (2019) 381-398.

Table II. Composition of the prepared CIC-AZL buccoadhesive tablets and their observed responses

\begin{tabular}{|c|c|c|c|c|c|c|c|}
\hline \multirow{2}{*}{ 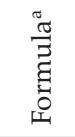 } & $\begin{array}{l}\text { Type of } \\
\text { polymer }\end{array}$ & $\begin{array}{l}\text { Polymer } \\
(\%)\end{array}$ & $\begin{array}{l}\text { Preparation } \\
\text { technique }^{\text {b }}\end{array}$ & Q2 (\%) & Q4 (\%) & Q8 (\%) & $\begin{array}{l}\text { Mucoadhesion } \\
\text { strength (g) }\end{array}$ \\
\hline & A & B & C & R1 & $\mathrm{R} 2$ & R3 & R4 \\
\hline $\mathrm{F} \# 1$ & $\mathrm{CMC} \mathrm{Na}$ & 5 & SM & $19.85 \pm 2.12$ & $29.54 \pm 2.11$ & $52.29 \pm 1.4$ & $30.23 \pm 1.33$ \\
\hline $\mathrm{F} \# 2$ & chitosan & 5 & SM & $11.84 \pm 3.12$ & $23.97 \pm 5.12$ & $40.78 \pm 2.55$ & $40.23 \pm 1.44$ \\
\hline $\mathrm{F} \# 3$ & Carbomer & 5 & SM & $8.13 \pm 2.48$ & $14.59 \pm 3.98$ & $30.6 \pm 2.22$ & $31.23 \pm 1.33$ \\
\hline $\mathrm{F} \# 4$ & CMC Na & 10 & SM & $10.76 \pm 1.44$ & $24.12 \pm 3.97$ & $48.32 \pm 3.11$ & $35.67 \pm 2.04$ \\
\hline $\mathrm{F} \# 5$ & chitosan & 10 & SM & $7.74 \pm 3.08$ & $19.92 \pm 4.22$ & $34.43 \pm 2.08$ & $44.18 \pm 2.63$ \\
\hline $\mathrm{F} \# 6$ & Carbomer & 10 & SM & $6.56 \pm 4.11$ & $12.11 \pm 2.44$ & $26.2 \pm 3.55$ & $35.56 \pm 1.93$ \\
\hline $\mathrm{F} \# 7$ & $\mathrm{CMC} \mathrm{Na}$ & 15 & SM & $8.04 \pm 4.12$ & $22.83 \pm 3.38$ & $42.12 \pm 2.56$ & $41.73 \pm 1.67$ \\
\hline $\mathrm{F} \# 8$ & chitosan & 15 & SM & $4.02 \pm 3.18$ & $10.23 \pm 4.34$ & $29.23 \pm 3.66$ & $48.99 \pm 1.87$ \\
\hline $\mathrm{F} \# 9$ & Carbomer & 15 & SM & $4.83 \pm 4.21$ & $9.23 \pm 3.68$ & $23.7 \pm 3.67$ & $41.11 \pm 2.06$ \\
\hline $\mathrm{F} \# 10$ & $\mathrm{CMC} \mathrm{Na}$ & 5 & LS & $60.86 \pm 2.77$ & $74.74 \pm 2.4$ & $95.09 \pm 3.23$ & $40.67 \pm 2.45$ \\
\hline F\#11 & chitosan & 5 & LS & $57.56 \pm 2.87$ & $70.71 \pm 2.98$ & $87.76 \pm 3.9$ & $27.67 \pm 2.17$ \\
\hline $\mathrm{F} \# 12$ & Carbomer & 5 & LS & $10.46 \pm 4.37$ & $23.56 \pm 4.54$ & $46.74 \pm 2.55$ & $30.22 \pm 2,64$ \\
\hline $\mathrm{F} \# 13$ & $\mathrm{CMC} \mathrm{Na}$ & 10 & LS & $45.99 \pm 3.49$ & $69.99 \pm 2.44$ & $88.79 \pm 4.41$ & $49.3 \pm 0.59$ \\
\hline $\mathrm{F} \# 14$ & chitosan & 10 & LS & $31.08 \pm 3.91$ & $61.43 \pm 2.5$ & $79.87 \pm 4.22$ & $30.1 \pm 1.77$ \\
\hline F\#15 & Carbomer & 10 & LS & $8.23 \pm 3.44$ & $16.23 \pm 3.69$ & $40.49 \pm 5.07$ & $35.2 \pm 2.15$ \\
\hline F\#16 & $\mathrm{CMC} \mathrm{Na}$ & 15 & LS & $24.57 \pm 2.54$ & $58.44 \pm 4.57$ & $85.44 \pm 3.97$ & $54.33 \pm 2.43$ \\
\hline $\mathrm{F} \# 17$ & chitosan & 15 & LS & $28.26 \pm 2.43$ & $56.44 \pm 3.02$ & $76.37 \pm 1.66$ & $32.14 \pm 1.58$ \\
\hline F\#18 & Carbomer & 15 & LS & $6.33 \pm 4.32$ & $13.4 \pm 3.38$ & $37.94 \pm 3.88$ & $41 \pm 1.78$ \\
\hline F\#19 & $\mathrm{CMC} \mathrm{Na}$ & 5 & WG & $47.45 \pm 1.77$ & $81.56 \pm 3.55$ & $98.3 \pm 4.11$ & $29.45 \pm 1.77$ \\
\hline $\mathrm{F} \# 20$ & chitosan & 5 & WG & $60.74 \pm 1.55$ & $75.91 \pm 2.58$ & $95.33 \pm 2.53$ & $30.12 \pm 2.54$ \\
\hline $\mathrm{F} \# 21$ & Carbomer & 5 & WG & $14.57 \pm 3.43$ & $26.36 \pm 1.69$ & $50.89 \pm 4.37$ & $33.29 \pm 1.43$ \\
\hline $\mathrm{F} \# 22$ & $\mathrm{CMC} \mathrm{Na}$ & 10 & WG & $42.45 \pm 3.14$ & $75.45 \pm 2.69$ & $94.56 \pm 3.11$ & $30.87 \pm 1.34$ \\
\hline $\mathrm{F} \# 23$ & chitosan & 10 & WG & $46.78 \pm 2.42$ & $65.74 \pm 2.69$ & $86.78 \pm 1.99$ & $33.23 \pm 2.24$ \\
\hline $\mathrm{F} \# 24$ & Carbomer & 10 & WG & $10.23 \pm 4.21$ & $18.92 \pm 5.02$ & $43.33 \pm 4.48$ & $34.54 \pm 2.4$ \\
\hline $\mathrm{F} \# 25$ & $\mathrm{CMC} \mathrm{Na}$ & 15 & WG & $38.4 \pm 4.26$ & $70.37 \pm 3.22$ & $90.84 \pm 3.76$ & $33.8 \pm 0.98$ \\
\hline $\mathrm{F} \# 26$ & chitosan & 15 & WG & $38.77 \pm 2.58$ & $60.42 \pm 1.97$ & $81.37 \pm 3.23$ & $35.9 \pm 1.39$ \\
\hline $\mathrm{F} \# 27$ & Carbomer & 15 & WG & $8.33 \pm 3.44$ & $15.44 \pm 4.13$ & $39.76 \pm 4.11$ & $35.17 \pm 2.05$ \\
\hline
\end{tabular}

${ }^{\mathrm{a}}$ Mean $\pm \mathrm{SD}(n=6), \mathrm{b} \mathrm{SM}-$ simple mix, LS - liquisol, WG - wet granulation.

After that, $1 \% \mathrm{Mg}$ stearate was mixed with each of the prepared dry powder mixtures for $2 \mathrm{~min}$; these mixtures were then compressed into a 150-mg core using a single-punch tablet machine (KORSCH XP1) with a 7-mm diameter.

\section{CIC preparation}

Each precompressed core was placed separately in the center of a 9.5-mm punch, then the turret was lowered, and the cup layer (50 mg ethyl cellulose) was added to the die 
containing the core and compressed again to attain CIC-AZL buccoadhesive tablets (Fig. S1 in Supplementary Material).

\section{Micromeritic formulation properties}

Precompression flow properties and compressibility of the CIC-AZL buccoadhesive tablets (including flowability, angle of repose, bulk density, tapped density, Carr's index and Hausner's ratio) were determined for AZL and blended powders of all the cores before compression (6).

\section{Characterizing the CIC-AZL buccoadhesive tablets}

Swelling index. - To determine the swelling index (SI) of CIC-AZL buccoadhesive tablets, the tablets were weighed individually $\left(m_{0}\right)$ and placed separately in Petri dishes containing distilled water at $\mathrm{pH} 6.4(n=3)$. Tablets were removed from the Petri dish each hour for $6 \mathrm{~h}$, and excess surface water was wiped carefully using filter paper. The swollen tablet was then reweighed $\left(m_{\mathrm{t}}\right)$, and the SI was calculated using the following formula (7):

$$
S I=\frac{m_{t}-m_{0}}{m_{0}} \times 100
$$

Mucoadhesive strength. - Bioadhesive forces of the prepared CIC-AZL buccoadhesive tablets were measured according to Kassem et al. (8). Briefly, freshly excised bovine buccal mucosa (obtained from a local slaughterhouse and stored in normal saline at $4{ }^{\circ} \mathrm{C}$ upon collection), were fixed on glass stages using cyanoacrylate adhesive. Prepared tablets were attached to the balance pan, and the glass stage was raised slowly until the tablet surface contacted the buccal mucosa. A preload of $50 \mathrm{~g}$ was applied over the balance pan above the tablet for 5 min to establish adhesion bonding between the tablet and buccal mucosa and was then removed. The preload weight and time were kept constant for all formulations. Increasing weights were applied until the tablet detached from the buccal mucosa. Minimum weight (in g) that detached the tablet from the membrane surface was recorded as a measure of mucoadhesive strength.

In vitro release study. - AZL release was performed using the USP Dissolution Tester, Apparatus II (rotating paddle, Erweka DT-700, Germany) at a rotation of $50 \mathrm{rpm}$. Experiments were carried out at $37 \pm 0.5^{\circ} \mathrm{C}$ in $900 \mathrm{~mL}$ of Sorenson phosphate buffer (pH 6.8; USP), containing $1 \%$ Tween- 80 as surfactant. The cup layer of the buccoadhesive tablet was attached to a glass disk using cyanoacrylate adhesive. The disk was placed at the bottom of the dissolution vessel. Samples were taken every hour for $8 \mathrm{~h}$, then filtered and analyzed for AZL content by the previously mentioned HPLC method. The dissolution test was performed using six tablets per formula (Fig. S2 in Supplementary Material).

Three time points were included in the in vitro dissolution specifications of the controlled-release tablets: an early time point to exclude dose dumping and/or characterize the initial dose (Q2 $=20-30 \%$ dissolved), a second time point to ensure compliance with the dissolution profile shape (Q4 $=40-60 \%$ dissolved) and a final time point to ensure that most of the drug was released (Q8 = more than $85 \%$ dissolved) (9). 


\section{Kinetic analysis of release data}

Release profiles were analyzed using the zero-order, first-order and Higuchi's squareroot equations $(10,11)$. The Hixson-Crowell model was also obtained to describe the release from systems with changes in the surface area and particle or tablet diameter (12).

\section{Ex vivo permeation study}

AZL permeation from the optimized formula (F\#16, chosen by the factorial design) through bovine buccal mucosa (freshly excised bovine buccal mucosa, $0.3-\mathrm{cm}$ thick, obtained from a local slaughterhouse and stored in normal saline at $4{ }^{\circ} \mathrm{C}$ upon collection after removing all fat and debris) was performed in triplicate using glass tubes opened from both sides with a 1.2-cm diameter. Each tablet was pressed on the mucosa for 30 seconds from the core side, and the tablet-loaded mucosa was stretched over an open end of the glass tube to form the donor chamber. Phosphate buffer $(4 \mathrm{~mL}, \mathrm{pH} 6.8)$ was added to the donor chamber. The tube was suspended on the side of the dissolution apparatus vessel so that the mucosa was just below the surface of $900 \mathrm{~mL}$ of phosphate buffer $(\mathrm{pH}$ 6.8) and was stirred at $50 \mathrm{rpm}$ at 37 $\pm 0.5^{\circ} \mathrm{C}$, with a diffusional surface area of $1.13 \mathrm{~cm}^{2}$. Five-milliliter samples were removed at set time intervals and replaced by equivalent amounts of fresh phosphate buffer ( $\mathrm{pH}$ 6.8). Permeated drug amounts were assayed using the previously described HPLC method.

\section{RESULTS AND DISCUSSION}

\section{High-performance liquid chromatography (HPLC) analyses}

Linearity was obeyed over a concentration range. The regression equation was computed, and the $R^{2}$ value was 0.998 . Relative standard deviations of the AZL solution concentrations were all below $2 \%$.

\section{Preformulation studies}

Differential scanning calorimetry (DSC). - The DSC thermogram of AZL showed a characteristic sharp melting endothermic peak at $123.6^{\circ} \mathrm{C}$, corresponding to the drug melting point. The DSC thermograms (in Supplementary Fig. S3) show the effect of mixing AZL with different excipients on the phase transition of the drug. Under experimental conditions, for all selected excipients, the observed endothermic peak in the physical mixture was similar to the peak of the drug. This indicates that the crystalline form of AZL, but not its physical form, exists in the physical mixture, indicating excipient incompatibility.

Fourier transform infrared spectroscopy (FTIR). - The infrared spectrum of AZL showed a band at $1069 \mathrm{~cm}^{-1}$ due to a C-N primary amine stretch, a band at $1101 \mathrm{~cm}^{-1}$ due to a secondary alcohol $\mathrm{C}-\mathrm{O}$ stretch, a band at $1486 \mathrm{~cm}^{-1}$ due to an aromatic ring stretch $\mathrm{C}=\mathrm{C}-\mathrm{C}$ bond, a band at $1652 \mathrm{~cm}^{-1}$ due to an aromatic nitro-oxy group and a band at $3315 \mathrm{~cm}^{-1}$ due to an aliphatic primary amine N-H stretch. The IR spectrum and PMs of AZL are shown in Supplementary Fig. S4. All AZL bands were preserved at the same positions, indicating that no interaction occurred between the drug and any of previous excipients. 
Screening the effects of different solubilizers on AZL solubility

Equilibrium solubilities of the AZL PMs in distilled water are shown in Table III. Effects of solubilizers on the solubility were improved by increasing their concentrations. The PMs of the AZL/Poloxamer 188 (1:1, 1:3, and 1:5, $\mathrm{m} / \mathrm{m})$ had the highest AZL solubilities, increasing from $1.4 \mu \mathrm{g} \mathrm{mL} \mathrm{m}^{-1}$ (pure AZL) to $49.3,56.7$, and $56.7 \mu \mathrm{g} \mathrm{mL}^{-1}$, respectively. These results were attributed to the wetting, solubilizing, and surface activity of Poloxamer 188. PMs with the other solubilizers increased slightly the solubility of AZL, which may have been due to their limited wetting and solubilizing properties. Thus, the AZL/Poloxamer 188 at a ratio of 1:3 was chosen for further investigation. Poloxamer 188 was also selected because of (1) its solid nature, which enabled the direct compression technique for the required tablets; (2) its low melting point $\left(m p=55^{\circ} \mathrm{C}\right)$, which allowed the application of the liquisol technique; and (3) its solubility in $95 \%$ ethanol, which allowed application of the wet granulation technique.

\section{Applying a $3^{3}$ full factorial design for buccoadhesive tablets}

The factorial design results are shown via prepared tablet characterizations. Statistical significance of the effects of the factors on the studied responses and their interactions is described.

\section{Micromeritic formulation properties}

Table IV shows the micromeritic formulation results. Formulations prepared using wet granulation showed good flowability, likely due to the change in particle size (13),

Table III. Effect of different solubilizers on the equilibrium solubility of AZL in distilled water

\begin{tabular}{lcc}
\hline Types of solubilizer & Drug/solubilizer ratio & Solubility $\left(\mu \mathrm{gL}^{-1}\right)$ \\
\hline Pure AZL & - & 1.4 \\
Cremophore RH 40 & $1: 1$ & 50 \\
& $1: 3$ & 53.7 \\
Poloxamer 188 & $1: 5$ & 53.7 \\
& $1: 1$ & 49.3 \\
Transcutol HP & $1: 3$ & 56.7 \\
& $1: 5$ & 56.7 \\
Labrasol & $1: 1$ & 7.1 \\
& $1: 3$ & 8.82 \\
PEG 6000 & $1: 5$ & 11.33 \\
& $1: 1$ & 43.3 \\
& $1: 3$ & 47.4 \\
& $1: 5$ & 55.7 \\
& $1: 1$ & 1.55 \\
& $1: 3$ & 1.61 \\
& $1: 5$ & 1.63 \\
\hline
\end{tabular}


A. A. Rashad et al.: Core-in-cup/liquisol dual tackling effect on azelnidipine buccoadhesive tablet micromeritics, in vitro release, and mucoadhesive strength, Acta Pharm. 69 (2019) 381-398.

Table IV. Micrometrics of the CIC-AZL buccoadhesive tablet formulation blended powder

\begin{tabular}{ccccccc}
\hline Formula & $\begin{array}{c}\text { Bulk } \\
\text { density } \\
\left(\mathrm{g} \mathrm{mL}^{-1}\right)\end{array}$ & $\begin{array}{c}\text { Tapped } \\
\text { density } \\
\left(\mathrm{g} \mathrm{L}^{-1}\right)\end{array}$ & $\begin{array}{c}\text { Carr's } \\
\text { index } \\
(\%)\end{array}$ & $\begin{array}{c}\text { Hausner's } \\
\text { ratio }\end{array}$ & $\begin{array}{c}\text { Angle } \\
\text { of repose } \\
\left({ }^{\circ}\right)\end{array}$ & $\begin{array}{c}\text { Flowability } \\
\left(\mathrm{g} \mathrm{s}^{-1}\right)\end{array}$ \\
\hline AZL & 0.333 & 0.476 & 30.042 & 1.4294 & 32.380 & 0.022 \\
F\# 1 & 0.574 & 0.709 & 18.998 & 1.234 & 30.891 & 3.252 \\
F\# 2 & 0.498 & 0.615 & 19.024 & 1.234 & 30.465 & 0.479 \\
F\# 3 & 0.450 & 0.556 & 19.028 & 1.235 & 36.253 & 1.000 \\
F \# & 0.561 & 0.710 & 20.906 & 1.264 & 34.813 & 1.587 \\
F\# 5 & 0.494 & 0.628 & 21.352 & 1.271 & 31.065 & 0.392 \\
F\# 6 & 0.479 & 0.612 & 21.650 & 1.276 & 38.016 & 0.800 \\
F\# 7 & 0.545 & 0.691 & 21.027 & 1.266 & 35.615 & 1.333 \\
F\# 8 & 0.481 & 0.614 & 21.582 & 1.275 & 32.221 & 0.324 \\
F\# 9 & 0.484 & 0.634 & 23.611 & 1.309 & 39.182 & 0.506 \\
F\# 10 & 0.523 & 0.619 & 15.395 & 1.181 & 28.009 & 4.000 \\
F\# 11 & 0.409 & 0.479 & 14.446 & 1.168 & 26.161 & 0.591 \\
F\# 12 & 0.420 & 0.498 & 15.662 & 1.185 & 29.394 & 0.800 \\
F\# 13 & 0.526 & 0.631 & 16.632 & 1.199 & 29.465 & 2.105 \\
F\# 14 & 0.403 & 0.484 & 16.659 & 1.199 & 26.980 & 0.444 \\
F\# 15 & 0.383 & 0.468 & 18.065 & 1.220 & 31.263 & 0.542 \\
F\# 16 & 0.527 & 0.636 & 17.224 & 1.208 & 31.686 & 1.600 \\
F\# 17 & 0.406 & 0.496 & 18.151 & 1.221 & 27.192 & 0.380 \\
F\# 18 & 0.371 & 0.456 & 18.504 & 1.227 & 32.485 & 0.416 \\
F\# 19 & 0.493 & 0.563 & 12.449 & 1.142 & 24.009 & 4.000 \\
F\# 20 & 0.429 & 0.490 & 12.285 & 1.140 & 25.836 & 0.642 \\
F\# 21 & 0.431 & 0.504 & 14.444 & 1.168 & 24.710 & 1.000 \\
F\# 22 & 0.476 & 0.562 & 15.339 & 1.181 & 25.463 & 1.724 \\
F\# 23 & 0.424 & 0.503 & 15.563 & 1.184 & 25.286 & 0.449 \\
F\# 24 & 0.383 & 0.460 & 16.586 & 1.198 & 25.303 & 0.888 \\
F\# 25 & 0.457 & 0.549 & 16.739 & 1.201 & 26.003 & 1.777 \\
F\# 26 & 0.423 & 0.510 & 16.980 & 1.204 & 25.134 & 0.400 \\
F\# 27 & 0.368 & 0.442 & 16.730 & 1.200 & 25.394 & 0.666 \\
\hline
\end{tabular}

while those prepared with the liquisol and simple mix techniques had reasonable flows. Carr's index determination revealed that all formulations prepared using the wet granulation and liquisol techniques had good flow, while those prepared with the simple mix technique showed fair flow. For the Hausner's ratio values, most formulations, except F\#9, had values of approximately 1.2, indicating low interparticle friction. This may have been 
due to the presence of Carbomer at a high concentration (15\%) in the simple mix technique, which gave poor flowability to the bulk. The results showed that Carr's index and Hausner's ratio increased as the polymer concentration increased, leading to poor flowability and compressibility. Hausner's ratio results correlated well with the Carr's index results, angle of repose, and flowability.

\section{Characterization of CIC-AZL buccoadhesive tablets and statistical analyses}

Swelling index

Buccoadhesive tablets require appropriate swelling behaviour for prolonged drug release and effective mucoadhesion. Being hydrogels, polymers swell readily when they contact a hydrated mucous membrane. Upon hydrating the tablets, the cores swelled with no change in cup appearance. Fig. 1 shows that increased concentrations of the three polymers in the formulations increased the swelling indices, which increased proportionally with the hydration rate as the tablets' weight increased. Formulations containing Carbomer P940 showed higher swelling indices than those containing CMC Na and chitosan. Swelling behaviour of Carbomer P940 was attributed to the uncharged - $\mathrm{COOH}$ group, which became hydrated, forming hydrogen bonds with the imbibing water and extending the polymer chain (14). The high water uptake by the $\mathrm{CMC}$ Na was due to repulsive forces of the negative charge of the carboxylate carried by $\mathrm{CMC} \mathrm{Na}\left(-\mathrm{COO}^{-} \mathrm{Na}^{+}\right)(15)$. Relatively fair swelling characteristics of chitosan were primarily related to its $\mathrm{pH}$-dependent solubility. Being basic in nature, chitosan is soluble in media with $\mathrm{pH}$ values lower than its $\mathrm{pK}_{\mathrm{a}}$ (approximately 6.3-6.5), leading to $100 \%$ ionization. While near its $\mathrm{pK}_{\mathrm{a}}$ (i.e., at $\mathrm{pH} 6.4$ in distilled water), $50 \%$ of chitosan molecules were ionized. The $-\mathrm{NH}_{3}{ }^{+}$group loses a proton and becomes $-\mathrm{NH}_{2}$, thus increasing the hydrophobicity of the hydrogel and hindering water penetration and swellability $(16,17)$. Thus, the decreased swelling indices of chitosan may have been due either to the increased degree of cross-linking leading to more difficult structural expansion or a restricted water influx due to formation of a viscous gel layer at the tablet and mucosa contact area.

\section{Effects of different factors on the studied responses}

The factorial design results (Table II) were analyzed individually using 2-factor interaction models (2FI) to describe the relationship between the response and the factors. Three-dimensional plots were used to screen the influence of factors on the responses (Fig. 3).

\section{Mucoadhesive strength}

Mucoadhesive strength indicates the ability of a tablet to adhere to the buccal mucosa. Such prolonged adhesion enables increased absorption and the possibility of formulating the desired controlled-release dosage form. The extent of mucoadhesion should depend on the surface distribution of the polymer and the degree of swelling. Mucoadhesiveness occurs first by forming a gel structure with increasing polymer chain flexibility to interact and entangle with the glycoprotein in the mucus and second via hydrogen bonding of the hydrophilic groups of the polymer with glycoproteins in the mucosal layer (18).

Mucoadhesive strength of the prepared formulations ranged from $27.67 \pm 2.2$ to $54.3 \pm$ $2.4 \mathrm{~g}$ (Table II). The mucoadhesive strength response was adequately modelled by $R^{2}=$ 0.983 , adjusted $R^{2}=0.945$ and predictive $R^{2}=0.807$. 

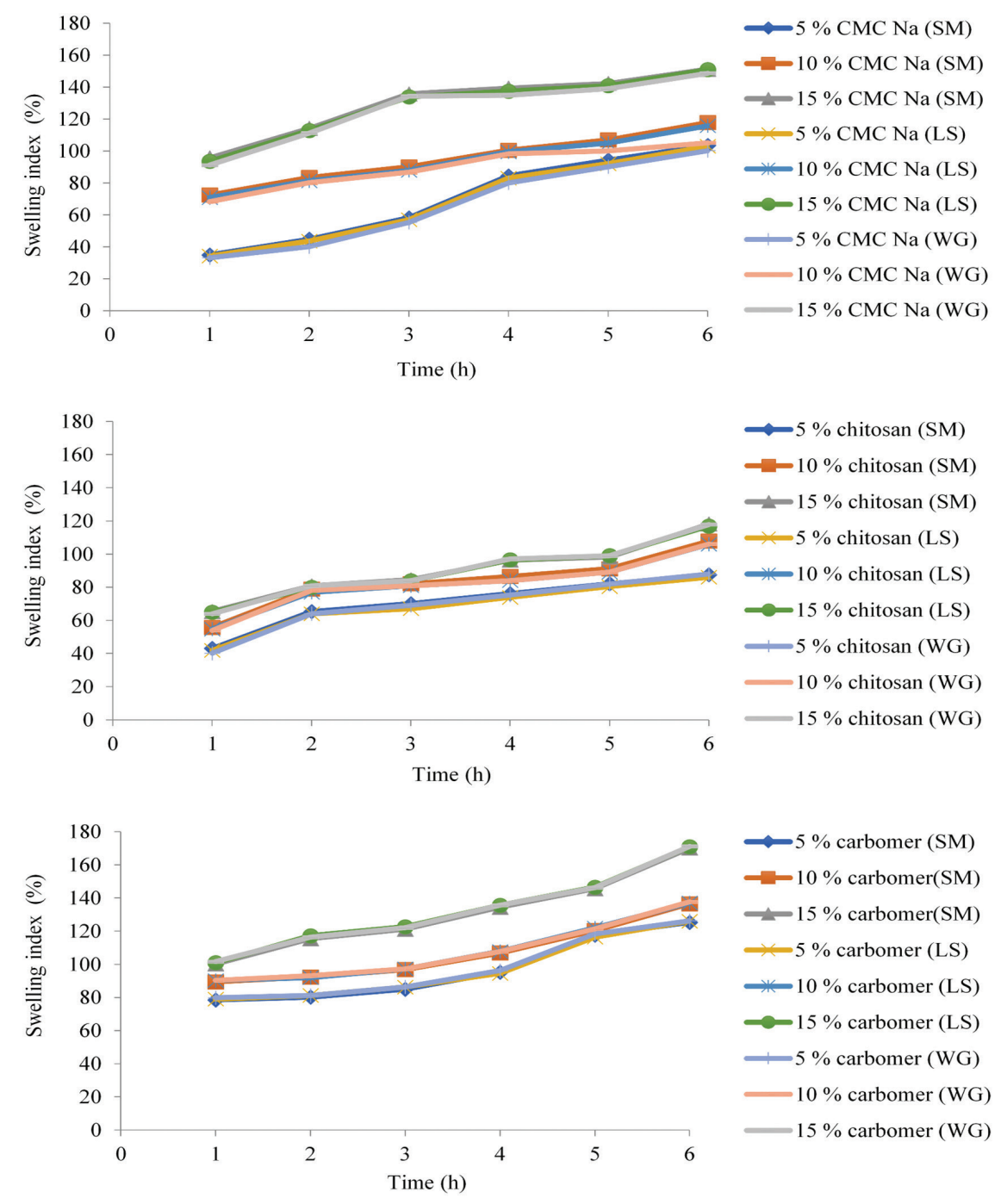

Fig. 1. Swelling indices of CIC-AZL buccoadhesive tablets using different polymers, different polymer concentrations and different preparation techniques; viz. SM - simple mix, LS - liquisol, WG - wet granulation $(n=3)$. Standard deviations are not shown in the figures for clarity of presentation.

Polymer type significantly affected the mucoadhesive strength. In the liquisol technique, CMC Na showed more pronounced mucoadhesive strength than the other polymers. The higher swelling indices of CMC Na played an important role in increasing the mucoadhesive strength due to the presence of hydrophilic groups. Hydration of these functional groups resulted in water entering the polymer network, leading to polymer expansion. Moreover, these hydrophilic groups formed hydrogen bonds with the mucosal 
layer (19). The performance of CMC Na may be related to the good balance between the available hydrogen bonding sites and an open expanded conformation (20). Conversely, in the simple mix technique, chitosan, a cationic polymer, showed more pronounced mucoadhesive strength than the other polymers. Mucoadhesion between chitosan and the buccal mucosa at a neutral $\mathrm{pH}$ resulted from a series of interactions: electrostatic attraction of the ionized amino groups with the sialic groups of mucin and hydrogen bonding due to the presence of free hydroxyl groups and hydrophobic effects (21). In contrast, chitosan showed reduced mucoadhesive strength in the liquisol and wet granulation techniques. This might be highly correlated with the decreased degree of ionization of chitosan molecules in both techniques. Studies have shown that the mucoadhesive properties of polymers containing ionizable groups are affected by the $\mathrm{pH}$ of the surrounding medium. Hence, the $\mathrm{pH}$-dependent solubility of chitosan was due to the difference between its $\mathrm{pK}$ and the $\mathrm{pH}$ of the buccal mucosa ( $\mathrm{pH}$ 6.8). Decreased ionization and chitosan aqueous solubility led to increased chitosan hydrophobicity and reduced cross-linking density, thus decreasing the degree of chitosan entanglement and electrostatic interaction with the mucosal layer. These results were consistent with the decreased levels in the swelling indices. Carbomer's mucoadhesive strength showed results similar to those of CMC Na due to the similar presence of repulsive forces of the carboxylate negative charge carried by both polymers. However, these similar results were statistically significant (ANOVA and post-hoc Least Significant Difference (LSD) test).

Increased polymer concentrations positively significantly affected mucoadhesive strength. Maximum values of mucoadhesive strength were attained at the highest levels of the three polymers. Higher concentrations may have had more penetrating chains than lower polymer concentrations, thus increasing the adhesive effect. For example, higher Carbomer concentrations upon exposure to moist surfaces lowered the microenvironmental $\mathrm{pH}$, thus causing increased mucoadhesion. Acidic environment favors excess uncharged - $\mathrm{COOH}$ groups, which form stronger hydrogen bonds with water and strengthen the mucoadhesive bond. These results are consistent with those of Bertram and Bodmeier (22), who investigated the effect of polymer solution concentrations used to prepare inserts on mucoadhesive strength. Increasing the chitosan concentration may produce more adhesive sites and polymer chains for interpenetration with mucin leading to increased mucoadhesive strength.

Preparation technique had the most pronounced effect on mucoadhesive strength, possibly due to the difference in each polymer's degree of hydration upon changing the preparation technique. Aside from chitosan, polymer molecules at the surface may be spaced sufficiently far apart from each other by all excipient molecules, allowing for rapid and complete hydration of the polymer structure, consequently producing high forces of adhesion following contact with the buccal mucosa (23). For chitosan, the direct and sole contact with Aeropril 300 (hydrophilic fumed silica) in the liquisol technique led to successful and stable adsorption on Aeropril (24). Kocun et al. (25) investigated chitosan adsorption on mica surfaces using atomic force microscopy. Images showed that the chitosan-adsorbed films were relatively smooth with a flat conformation. These results are closely correlated with the chitosan-containing tablet micromeritics using the liquisol technique, which showed a high degree of flowability compared to its counterparts. This might be attributed to the formation of smooth adsorbed films on the Aeropril surface enhancing the tablet flow properties. Such adsorption interaction was clearly reduced in the simple mix and wet granulation due to other interfering excipients, which decreased direct contact between chitosan and Aeropril. 


\section{In vitro release studies}

The Q2 \% response was adequately modelled by $R^{2}=0.978$, adjusted $R^{2}=0.928$ and predictive $R^{2}=0.746$. The Q4 \% response was adequately modelled by $R^{2}=0.998$, adjusted $R^{2}=0.994$ and predictive $R^{2}=0.979$. The Q8 \% response was adequately modelled by $R^{2}=$ 0.9996 , adjusted $R^{2}=0.9985$ and predictive $R^{2}=0.995$.

Dissolution profiles for all formulations are represented in Fig. 2, and the Q2 \%, Q4 \%, and Q8 \% responses are shown in Table II. The drug release mechanism from swellable
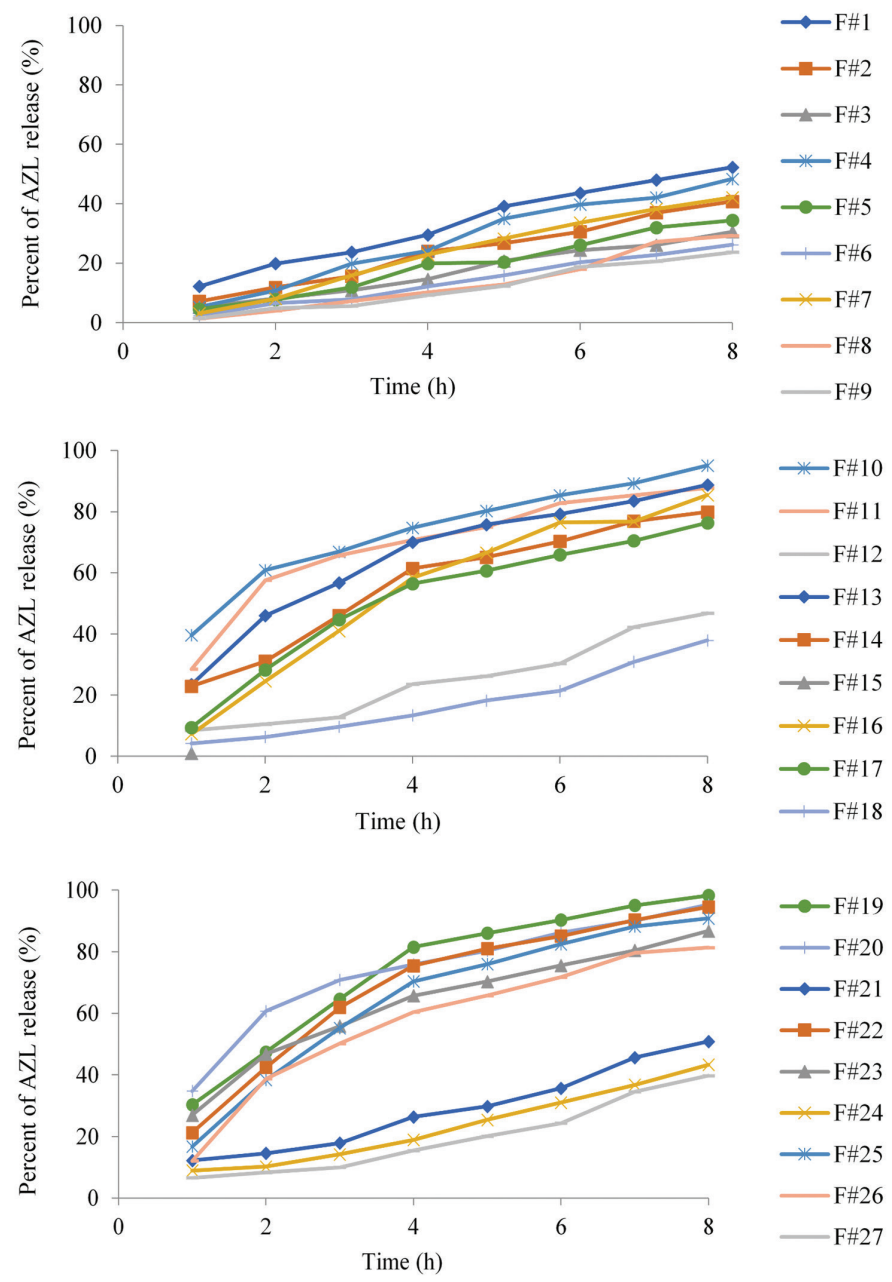

Fig. 2. In vitro release profile of CIC-AZL buccoadhesive tablets using different polymers, different polymer concentrations and different preparation techniques carried out in $900 \mathrm{~mL}$ of Sorenson phosphate buffer pH 6.8 at $50 \mathrm{r} / \mathrm{min}$ and $37 \pm 0.5{ }^{\circ} \mathrm{C}$ containing $1 \%$ Tween $80(n=6)$. Standard deviations are not shown in the figures for clarity of presentation. 

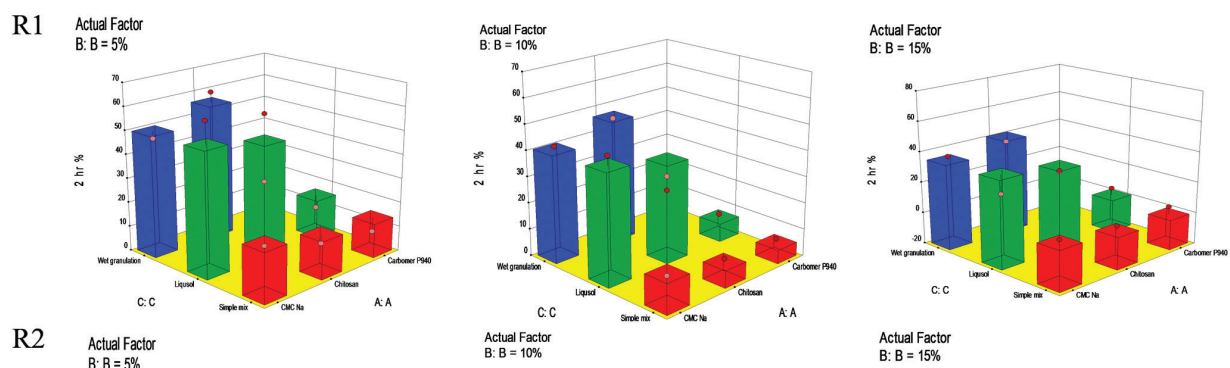

$\mathrm{R} 2$
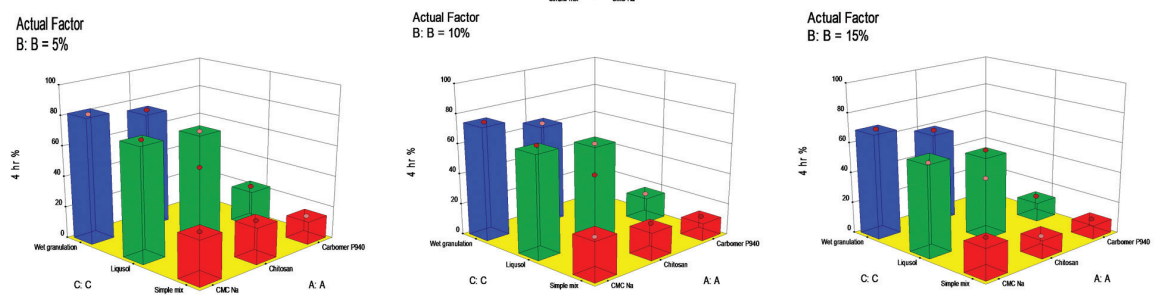

R3

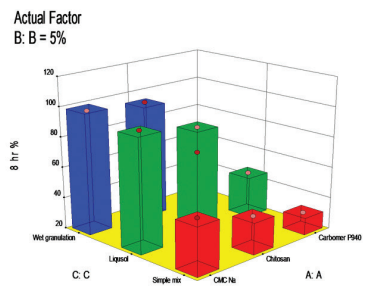

Actual Factor
$B: B=10 \%$

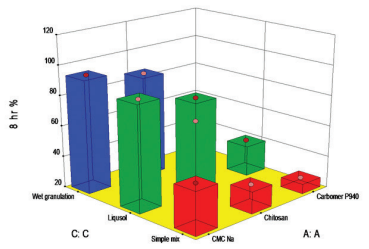

Actual Factor
$B: B=15 \%$

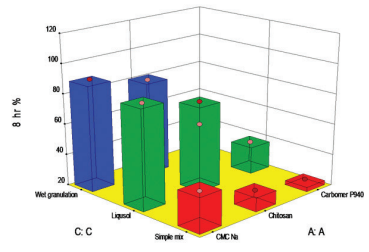

R4
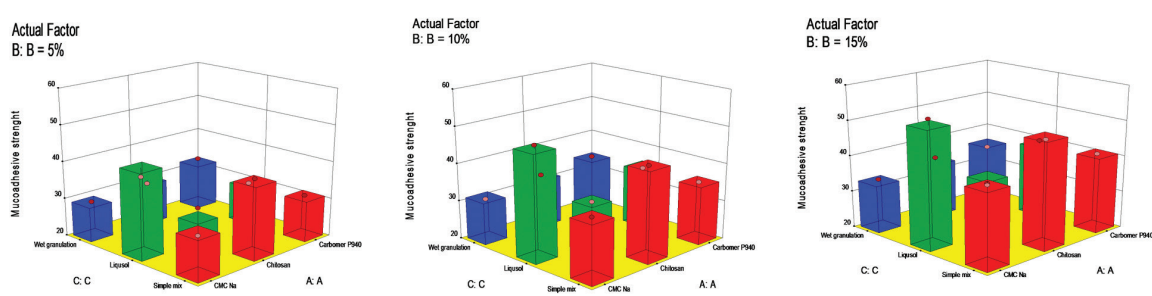

Fig. 3. 3D plot of AZL in CIC-AZL tablets demonstrating the effect of the studied factors on the response; R1: \% drug released after 2 h, R2: \% drug released after 4 h, R3: \% drug released after 8 h, and R4: mucoadhesive strength.

matrices could be compared via several physicochemical phenomena. Water uptake, gel layer formation, and polymeric chain relaxation were primarily involved in the drug release modulation (14). Initial high drug release was observed in some formulations at the $2 \mathrm{~h}$ time point (formulations prepared with 5 and $10 \%$ of both CMC Na and chitosan) and was reduced at subsequent time points. This may have been due to the initial rapid flux of the drug from the particles to the dissolution medium, which was reduced with time.

Polymer type significantly affected the percentage of the drug released at different time intervals (2, 4, and $8 \mathrm{~h}$ ). Tablets containing Carbomer P940 failed to control drug release over the $8 \mathrm{~h}$ release period, since no more than $50 \%$ of the drug was released after $8 \mathrm{~h}$ from all the formulations prepared using different techniques and concentrations. This 
could be attributed to the gelation of the matrix after the initial wetting, in addition to subsequent formation of more viscous gel layers on the tablet, which reduced the elution rate of the dissolved drug from the tablet core. Rapid AZL release from tablets containing CMC Na may have been due to the low viscosity (50-200 mPa s) of CMC Na, which led to high swellability and rapid erosion of the diffusion gel layer (26). Increasing the time led to sufficient hydration and polymer network swelling. Complete opening of the interpolymeric pores occurred indefinitely when in contact with water, eventually dissolving completely. Chitosan is soluble in acidic media, and the chitosan surface was eventually exposed to a neutral $\mathrm{pH}$ environment $(\mathrm{pH}=6.8)$ where it was rendered insoluble and thus showed a decreased dissolution profile compared to that of CMC Na (27).

Polymer concentration significantly negatively affected the percentage of drug released at different time intervals $(2,4$, and $8 \mathrm{~h})$, increasing the polymer concentration and retarding the AZL release. At lower levels, tablet hydration loosened the matrix at the surface, enabling water to diffuse into the core. These higher polymer levels led to significant decreases in drug release. This might be attributed to the increased viscosity and swellability of the gel layer, which slowed the erosion and increased the path that the drug had to traverse to be released, thus lessening its release (28). Polymers may also have had incomplete water access and were incompletely hydrated upon contact with the dissolution medium (23).

The preparation technique affected the release profile significantly. Tablet formulations prepared using the simple mix technique failed to give the required drug amount at the 2, 4, 6 and $8 \mathrm{~h}$ time points, since no more than $50 \%$ of the drug was released after $8 \mathrm{~h}$. These results might be due to the presence of Poloxamer 188 in solid form in the physical mixture. Formulations prepared using the wet granulation technique showed the highest drug release for the three polymers at different concentrations, since the presence of Poloxamer 188 in the dissolved or melted form showed significantly enhanced dissolution characteristics compared to those of PMs because Poloxamer 188 increased the dissolution rate by combined actions of the surface activity, solubilization and wetting effect. The dissolution rate increased with time due to increased surface area. The liquisol technique showed a medium-release profile, where the presence of the drug in the dissolved form improved the release profile while complying with the standards of the controlled-release dosage form (9). These results are consistent with those of many studies that used the liquisol technique to enhance the solubility and dissolution of insoluble drugs (29). It is likely that liquisol enhancement occurred because the drug was completely dissolved in Poloxamer 188 in a molecularly dispersed state. Thus, the drug had an increased surface area available for release. At the interface between the liquisol particles and the medium, the liquid vehicle acts as a cosolvent, increasing the aqueous solubility of the drug. Poloxamer 188 acted as an active surface agent improving the liquisol particle wetting. The wet granulation technique showed a marked increase in the dissolution profile compared to most liquisol formulations, possibly because the increased diffusional path length of the drug may paradoxically delay such release. Further, the thick gel layer formed on the swollen liquisol core surface can control water penetration. Diffusion of the drug through the swollen polymer may also be retarded because of clogged pores (30).

\section{Kinetic analysis of the release data}

The results (Table SI in Supplementary Material) showed that F\#3 F\#11, F\#12, F\#16, F\#19, F\#20, F\#21, F\#22, F\#23, F\#24, F\#25, F\#26 and F\#27 followed zero-order kinetics, in which 
the same amount of the drug was released per unit of time independently of drug concentration. Release data from $\mathrm{F} \# 4$ followed a diffusion-controlled mechanism as per the Higuchi model, while the remaining AZL formulations followed first-order kinetics. The regression coefficient of the optimized formulation (F\#16) was calculated from the Hixson-Crowell equation. The $r^{2}$ value of F\#16 was approximately equal to the $r^{2}$ values of the Higuchi kinetics, indicating unidirectional drug release.

\section{Determining the optimized formulation}

After generating final equations relating the dependent and independent variables, the studied responses were optimized. Numerical optimization searched the design space using the final models created for each studied response to determine the levels of factors that met the constraints set for the different responses. F\#16 prepared by the liquisol technique, containing $15 \% \mathrm{CMC} \mathrm{Na}$, was chosen with an overall desirability of 0.953 . The value of the desirability indicates that the chosen formulations satisfied the constraints given in Table I. F\#16, which contained $15 \%$ CMC Na and was prepared using the liquisol technique, showed the highest mucoadhesive strength of $54.33 \mathrm{~g}$ and had the optimal release profile, in which $24.57 \%$ of the drug was released after $2 \mathrm{~h}, 58.44 \%$ after $4 \mathrm{~h}$, and 85.44 $\%$ after $8 \mathrm{~h}$. These results complied with the required dissolution profile.

\section{Ex vivo permeation study}

AZL permeation from F\#16 was slow and steady as shown in Fig. 4, where $81.44 \% \pm$ 0.34 of AZL permeated the buccal membrane in $8 \mathrm{~h}$ with a flux of $0.193 \mathrm{mg} \mathrm{h}^{-1} \mathrm{~cm}^{-2}$ and

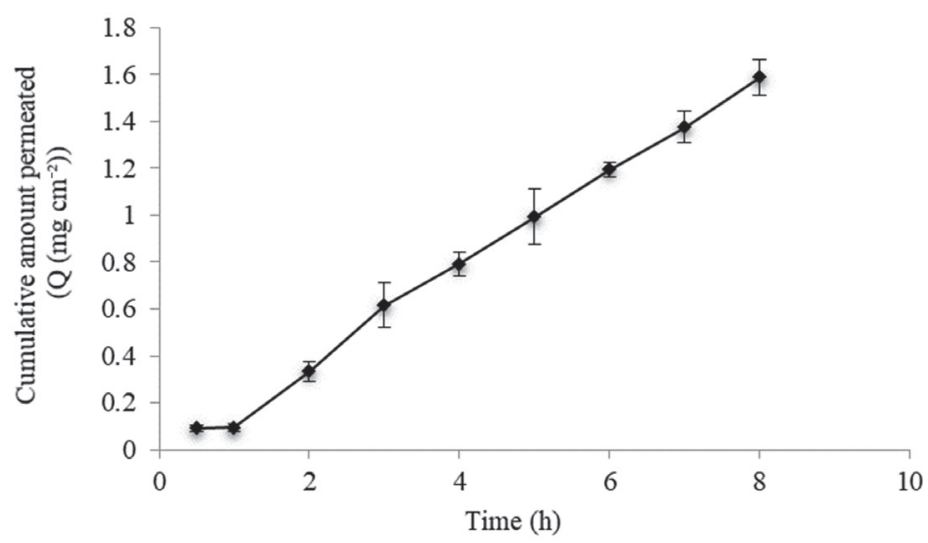

Fig. 4. Ex vivo permeation study of the optimized CIC-AZL buccoadhesive tablet (F\#16 which contained $15 \%$ CMC Na prepared using the liquisol technique), carried out in $900 \mathrm{~mL}$ of Sorenson phosphate buffer $\mathrm{pH} 6.8$ at $50 \mathrm{r} / \mathrm{min}$ and $37 \pm 0.5^{\circ} \mathrm{C}(n=3)$.

permeability coefficient $\left(K_{\mathrm{p}}\right)$ of $0.024 \mathrm{~cm} \mathrm{~h}^{-1}$. The lag time was short $(0.137 \mathrm{~h})$. Successful increase in solubility and dissolution rate attained by the dual effects of CIC/liquisol are believed to be the main reasons for the increased permeation rates. 


\section{CONCLUSIONS}

This research evaluated the key factors affecting the in vitro release studies and mucoadhesive strength to enhance AZL bioavailability. The poor aqueous solubility and firstpass metabolism of AZL pose the main challenges to drug delivery and were successfully overcome by formulating a liquisol CIC-AZL buccoadhesive tablet. The liquisol core was prepared and placed in CIC buccoadhesive tablets as a simple, easy, scalable and effective method of enhancing the dissolution profile of AZL. The ex vivo permeation study showed slow and steady AZL permeation. Therefore, F\#16 composed of $15 \%$ CMC Na and prepared using the liquisol technique to form a CIC buccoadhesive tablet is a promising drug delivery system.

\section{REFERENCES}

1. D. Prabhakar, J. Sreekanth and K. N. Jayaveera, Development and evaluation of transdermal patches of azelnidipine, Int. J. Pharm. Pharm. Sci. 5 (2013) 805-810.

2. K. Wellington and L. J. Scott, Azelnidipine, Drugs 63 (2003) 2613-2621; https://doi.org/10.2165/00003495200363230-00004

3. M. Kharwade and M. Sneha, A review on pioneering technique - liquisolid compact and applications, Res. J. Pharm. Biol. Chem. Sci. 6 (2015) 220-227.

4. D. Modi, P. Amaliyar, Y. Kalal, B. Gangadia, S. Chaudhary, K. Sanghvi, H. Shah and D. Y. Sen, Novel approach in compressed-coated tablet dosage form: Core-in-cup (in lay) tablet with geometrically altered drug delivery concept, Brit. Bio. Bull. 1 (2013) 90-102.

5. J. K. Patel and N. K. Patel, Validated stability-indicating RP-HPLC method for the simultaneous determination of azelnidipine and olmesartan in their combined dosage form, Sci. Pharm. 82 (2014) 541-554; https://doi.org/10.3797/scipharm.1312-14

6. British Pharmacopeia 2015, TSO, London 2015.

7. B. Parodi, E. Russo, G. Caviglioli, S. Cafaggi and G. Brignardi, Development and characterization of a buccoadhesive dosage form of oxycodone hydrochloride, Drug Dev. Ind. Pharm. 22 (1996) 445450; https://doi.org/10.3109/03639049609069353

8. M. A. A. Kassem, A. N. ElMeshad and A. R. Fares, Enhanced bioavailability of buspirone hydrochloride via cup and corebuccal tablets: Formulation and in vitro/in vivo evaluation, Int. J. Pharm. 463 (2014) 68-80; https://doi.org/10.1016/j.ijpharm.2014.01.003

9. European Medicines Agency, Guideline on quality of oral modified release products, 2012.

10. T. Higuchi, Mechanisms of sustained action medication, theoretical analysis of the rate of release of solid drugs dispersed in solid matrices, J. Pharm. Sci. 52 (1963) 1145-1149; https://doi.org/10.1002/ jps. 2600521210

11. R. W. Korsmeyer, R. Gurny, E. Doelker, P. B. Nikolaos and A. Peppas, Mechanism of solute release from porous hydrophilic polymers, Int. J. Pharm. 72 (1983) 1189-1191; https://doi.org/10.1016/03785173(83)90064-9

12. S. Dash, P. N. Murthy, L. Nath and P. Chowdhury, Kinetic modeling on drug release from controlled drug delivery systems, Acta Pol. Pharm. 67 (2010) 217-223.

13. J. Wu, H, Ho and M. Sheu, Influence of wet granulation and lubrication on the powder and tableting properties of codried product of microcrystalline cellulose with beta-cyclodextrin, Eur. J. Pharm. Biopharm. 51 (2001) 63-69.

14. R. J. Dias, S. S. Sakhare and K. K. Mali, Design and development of mucoadhesive acyclovir tablet, Iran J. Pharm. Res. 8 (2009) 231-239.

15. Y.-C. Chen, H.-O. Ho, D.-Z. Liu, W.-S. Siow and M.-T. Sheu, Swelling/floating capability and drug release characterizations of gastroretentive drug delivery system based on a combination of hy- 
droxyethyl cellulose and sodium carboxymethyl cellulose, PloS. one. 10 (2015) e0116914; https://doi. org/10.1371/journal.pone.0116914

16. M. Mucha, Rheological characteristics of semi-dilute chitosan solutions, Macromol. Chem. Phys. 198 (1997) 471-484; https://doi.org/10.1002/macp.1997.021980220

17. A. Martínez-Ruvalcaba, J. C. Sánchez-Díaz, F. Becerra, L. E. Cruz-Barba and A. González-Álvarez, Swelling characterization and drug delivery kinetics of polyacrylamide-co-itaconic acid/chitosan hydrogels, Express Polym. Lett. 3 (2009) 25-32; https://doi.org/10.3144/expresspolymlett.2009.5

18. J. W. Lee, J. H. Park, J. R. Robinson, Bioadhesive-based dosage forms: the next generation, J. Pharm. Sci. 89 (2000) 850-866; https://doi.org/10.1002/1520-6017(200007)89:7<850::aid-jps2>3.3.co;2-7

19. J. Akbari, M. Saeedi, K. Morteza-Semnani, B. Zarrabi, S. S. Rostamkalaei and H. R. Kelidari, The effect of Plantago major seed mucilage combined with carbopol on the release profile and bioadhesive properties of propranolol HCl buccoadhesive tablets, Pharm. Biomed. Res. 2 (2016) 84-100; https://doi.org/10.18869/acadpub.pbr.2.2.84

20. F. Madsen, K. Eberth and J. D. Smart, A rheological examination of the mucoadhesive/mucus interaction: the effect of mucoadhesive type and concentration, J. Control. Release 50 (1998) 167-178.

21. V. V. Khutoryanskiy, Advances in mucoadhesion and mucoadhesive polymers, Macromol. Biosci. 11 (2011) 748-764; https://doi.org/10.1002/mabi.201000388

22. U. Bertram and R. Bodmeier, In situ gelling, bioadhesive nasal inserts for extended drug delivery: in vitro characterization of a new nasal dosage form, Eur. J. Pharm. Sci. 27 (2006) 62-71; https://doi. org/10.1016/j.ejps.2005.08.005

23. M. S. Surapaneni, S. K. Das and N. G. Das, Effect of excipient and processing variables on adhesive properties and release profile of pentoxifylline from mucoadhesive tablets, Drug Dev. Ind. Pharm. 32 (2006) 377-387; https://doi.org/10.1080/03639040500519367

24. A. Tiraferri, P. Maroni, D. Caro Rodriguez and M. Borkovec, Mechanism of chitosan adsorption on silica from aqueous solutions, Langmuir 30 (2014) 4980-4988; https://doi.org/10.1021/la500680g

25. M. Kocun, M. Grandbois and L. A. Cuccia, Single molecule atomic force microscopy and force spectroscopy of chitosan, Colloids. Surf. B. 82 (2011) 470-476; https://doi.org/10.1016/j.colsurfb.2010.10.004

26. H. Abdelkader, O. Y. Abdalla and H. Salem, Formulation of controlled-release baclofen matrix tablets: Influence of some hydrophilic polymers on the release rate and in-vitro evaluation, AAPS. Pharm. Sci. Tech. 8 (2007) 156-166; https://doi.org/10.1208/pt0804100

27. S. Prabhu, N. Kanthamneni and C. Ma, Novel combinations of rate-controlling polymers for the release of leuprolide acetate in the colon, Drug Deliv. 15 (2008) 119-125; https://doi. org/10.1080/10717540801905157

28. S. Agarwal and R. S. R. Murthy, Effect of different polymer concentration on drug release rate and physicochemical properties of mucoadhesive gastroretentive tablets, Indian J. Pharm. Sci. 77 (2015) 705-714; https://doi.org/10.4103/0250-474x.174993

29. C. Naveen, S. Nalini and R. R. Tadikonda, Use of the liquisolid compact technique for improvement of the dissolution rate of valsartan, Acta. Pharm. Sin. B. 2 (2012) 502-508; https://doi.org/10.1016/j. apsb.2012.07.005

30. V. Vigoreaux and E. S. Ghaly, Fickian and relaxational contribution quantification of drug release in a swellable hydrophilic polymer matrix, Drug Dev. Ind. Pharm. 20 (1994) 2519-2526; https://doi. org/10.3109/03639049409042655 